\title{
Freqüência de imunofenótipos aberrantes em leucemias agudas
}

\author{
The frequency of aberrant immunophenotypes in acute leukemias
}

\author{
Mariana Emerenciano', Yomaira Bossa', Crisiane W Zanrosso', Dora Maria Alencar², Mércia Mendes Campos', Jane Dobbin ${ }^{3}$, Kadma Carriço ${ }^{3}$, \\ Maria S Pombo de Oliveira'
}

\section{Resumo}

As leucemias agudas (LA) são doenças heterogêneas, com características clínicas, morfológicas, imunológicas e moleculares distintas. Por meio destas particularidades, as leucemias são classificadas em seus diversos subtipos biológicos. N este estudo, nós analisamos a freqüência de fenótipos aberrantes (FA) em pacientes com leucemias, provenientes de diferentes regiões do Brasil, com o objetivo de avaliar estes FAs nos estudos futuros sobre doença residual mínima. Análises por citometria de fluxo multiparamétrica permitem o conhecimento da diferenciação hematopoética normal, bem como o perfil da diferenciação nas leucemias. 0 painel original com anticorpos monoclonais (AcM o) consistiu de marcadores distribuídos em associações ou testes com simples marcações. Entre os casos leucêmicos analisados neste estudo, 8,33\% correspondem a amostras cujos blastos expressam FA. A leucemia linfoblástica aguda (LLA) foi responsável por $46,67 \%$ dos casos com anomalia de fenótipo, enquanto a leucemia mielóide aguda (LM A) atendeu por $53,33 \%$ dos casos com FA. Entre os casos de LLA, o fenótipo mais freqüente foi $C D 10+/ C D 13+$. Em relação a $L M A$, a expressão do $C D 7$ foi predominante, principalmente entre os subtipos M 0, M 1 e M 2, seguida do CD56+ e do CD 19+. As análises nos indicaram pequenas diferenças nas freqüências de FAs nos diferentes estados brasileiros. No entanto, estas diferenças devem ser valorizadas na aplicação de painel de AcM o, nas pesquisas de doença residual mínima em nossa população.

Palavras-chave: Leucemia aguda; Imunofenotipagem; D oença residual mínima.

1- Divisão de M edicina Experimental - INCA, Rio de Janeiro.

2. Sociedade de O ncologia da Bahia e H ospital São Rafael, Salvador, Bahia.

3- Serviço de H ematologia do H ospital do Câncer I - IN CA, Rio de Janeiro.

Endereço para correspondência: M.S.P.O - Instituto N acional de Câncer - Coordenadoria de Pesquisa - Divisão de M edicina

Experimental - Rua André Cavalcanti, 37- Centro. CEP: 20231-050. Rio de Janeiro - RJ, Brasil. 


\begin{abstract}
Acute leukemias $(A L)$ are biologically diverse diseases in terms of their cellular phenotypes and molecular features. According to these characteristics, AL may be classified into distinct subtypes that may help to uncover the etiopathological mechanisms involved. In this study, we determined the frequency of aberrant immunophenotypes among patients with $\mathrm{AL}$ who came from different Brazilian regions. We attempt than to provide new data about the subject and to consider about the possible interferences of the aberrant phenotypes on the disease outcome. M ultiparameter flow cytometric analyses allowed us to acquire antigens observed during normal hematopoiesis, which reflect the $A L$ in their malignant form. The original panel with monoclonal antibodies ( $M O A b$ ) included markers in dual color associations or simple forms. Among patient's samples with $\mathrm{AL}, 8.33 \%$ were due to blasts with aberrant antigen expression. According to this study, $46.67 \%$ of acute lymphoblastic leukemia (ALL) cases and $53.33 \%$ of acute myeloid leukemia (AML) cases have aberrant expression of a single antigen associated with another cell lineage, mostly CD 13 in ALL (CD 10+) and CD 7 in AM L (M 0-M 2). In conclusion, the aberrant immunophenotypes frequencies determined among the $A L$ cases did not present significant differences between the different Brazilian states. H owever, prospective studies are required to understand whether these markers modify the disease outcome in our patients and their value in the detection of minimal residual disease.
\end{abstract}

Key words Acute leukemia; Immunophenotyping; M inimal residual disease.

\section{INTRODUÇÃO}

A leucemia aguda (LA) é uma doença bastante heterogênea de progresso rápido que afeta a maior parte das células hematopoéticas ainda não diferenciadas. Para 0 entendimento deste vasto grupo de entidades patológicas, são necessárias diferentes abordagens analíticas e associações de diversas modalidades de estudos. O s blastos leucêmicos na leucemia mielóide aguda (LMA) e na leucemia linfoblástica (LLA) expressam antígenos de diferenciação mielóide ou linfóide, respectivamente, com um padrão bem definido, e com critérios já bem estabelecidos ${ }^{1}$.

A definição de fenótipos aberrantes está freqüentemente associada às leucemias identificadas com, i) co-expressão de marcadores que raramente ou nunca são encontrados simultaneamente na diferenciação hematopoética normal, ii) com a superexpressão de um marcador específico de linhagem celular, ou iii) com a ausência de um marcador, que configura assincronia maturativa da célula ${ }^{2-7}$.

Estudos imunológicos e moleculares mostram que muitas LAs podem apresentar características de mais de uma linhagem celular. Estas observações permitiram que fossem então descritas duas categorias de LA que demonstram 'infidelidade de linhagem', são elas: LLA com antígenos associados à linhagem mielóide (LLA $\mathrm{My+)}$ e LM A com antígenos associados à linhagem linfóide $(L M A L y+)^{8}$. 0 critério recomendado para a classificação destes subgrupos leucêmicos sugere parâmetros que possibilitam a detecção da expressão antigênica ambígua (tabela 1). No entanto, é importante que se faça uma distinção entre leucemia bifenotípica aguda e os casos de LLA e LM A com expressão aberrante de marcadores de outras linhagens, devido às diferenças de condutas terapêuticas. Assim, é recomendável que ao estudar os casos com marcadores aberrantes, seja incluído o sistema de pontuação sugerido pelo grupo EGIL e a utilização de múltiplos AcM o com análise multiparamétrica através de citometria de fluxo (tabela 2).

O s mecanismos pelos quais ocorre a expressão de FA no desenvolvimento das LAs ainda permanecem obscuros, no entanto, já é possível estabelecer associações entre estas expressões incomuns e outras características biológicas da doença, como as associações com translocações cromossômicas e fatores prognósticos. Além disso, os imunofenótipos aberrantes são sabidamente instrumentos de grande importância na detecção de doença residual mínima (DRM).

Em muitos casos, existe associação entre FA e genes anômalos. Esta correlação pode ser exemplificada pela $L L A$ pró- $B$, com gene $A F 4 / M L L$, que tipicamente apresenta os marcadores celulares CD19+, CD 34+, T dT +, CD 10- freqüentemente associados a CD $15+$ e/ ou CD 13, além de N G $2^{1}$. N os casos de LLA com o gene TEL/AM L1, o fenótipo apresentado é exclusivo para precursores de célula $B$, com o fenótipo CD 19+, CD 34t, TdT + e CD 10t. Estes casos como demonstrado por diferentes estudos, freqüentemente são positivos para os marcadores mielóides CD 33 e/ou 
CD 13 (9-11). 0 s objetivos deste trabal ho foram detectar os FAs, descrever suas freqüências em $L A s$, e correlacionar os resultados obtidos com dados demográficos e subtipos celulares.

Tabela 1 - Diferentes aspectos Imunofenotípicos das leucemias agudas com aberrações de fenótipo

LLA $(M+)$ Células precursoras $B^{a}$
Células leucêmicas são:
1. CD79a ${ }^{+}$ou clg ì ${ }^{+}$ou $\mathrm{CD} 19^{+}$e CD22 ${ }^{+}$
2. CD3
3. MPO
4. CD13, CD15, CD33, CD65 ou CD 14+

\section{$\operatorname{LLA}(M+)$ Linhagem $T^{a}$}

Células leucêmicas são:

1. $\mathrm{CD}^{+}$e $\mathrm{CD}^{+}$(membrana/citoplasma)

2. $C D 79 a$

3. MPO

e expressam CD13, CD15, CD33, CD65 ou CD14

a Todos os critérios devem estar em acordo.

b Q uando os marcadores incidem na mesma célula blástica, trata-se de BAL, se em "pool" de células separadas, trata-se de leucemia bilineal ou mista. M odificado de Campana e cols., 2000.

Tabela 2 - Sistema de pontuação para a definição das leucemias bifenotípicas agudas

\begin{tabular}{c|c|c|c}
\hline Pontos & Linhagem B & Linhagem T & Linhagem mielóide \\
\hline 2 & $\begin{array}{c}\text { CD79a } \\
\text { cit IgM } \\
\text { cit CD22 }\end{array}$ & $\begin{array}{c}\text { CD3 }(\mathrm{c} / \mathrm{m}) \\
\text { anti-TCR } \alpha / \beta \\
\text { anti-TCR } \gamma / \delta\end{array}$ & $\begin{array}{c}\text { anti-MPO } \\
\text { (anti-lisozima) }\end{array}$ \\
\hline 1 & CD19 & CD2 & CD13 \\
& CD10 & CD5 & CD33 \\
& CD20 & CD8 & CDw65 \\
& CD10 & CD10 & CD14 \\
\hline 0,5 & CD24 & CD7 & CD15 \\
& & CD1 & CD64 \\
& & & CD117 \\
\hline
\end{tabular}

Fonte: EGIL, 1995.

\section{MATERIAL E MÉTODOS}

AM OSTRAS. Foram incluídas nesta análise, amostras de pacientes portadores de LA, procedentes de diversos estados brasileiros, sem restrição de idade, raça ou sexo e isentos de tratamento quimioterápico prévio. Amostras de medula óssea (M O) e/ou sangue
Células leucêmicas são:

1. $\mathrm{MPO}^{+}$ou no mínimo 2 outros marcadores como CD117+, CD33+ ou CD13+

2. $\mathrm{CD}^{-}$

3. CD79a-

4. CD2, CD5, CD7, CD19, CD22 ou CD56+ Leucemias com linhagens mistas ou Bifenotípicas Células leucêmicas co-expressam:

1. MPO e CD79a ou clg ì ou

2. $\mathrm{MPO}$ e CD3 ou

3. $\mathrm{cCD} 3$ e clg ì

\section{$M A L+{ }^{a}$}


SEPARAÇÃO CELULAR E IM UN OFEN OTIPAGEM . A etapa inicial consistia na separação celular para obtenção das células mononucleares, baseada em gradiente de centrifugação através de Ficoll-H ypaqueH ystopaque ${ }^{\circledR}$ (densidade 1077). D epois da retirada das células no anel de interfase do tubo contendo as células mononucleares, avaliava-se a viabilidade celular para então proceder a imunofenotipagem. As células mononucleares foram colocadas em tubos apropriados, para leitura no citômetro de fluxo, em concentração de 106 células e incubadas durante 30 minutos com $10 \mu \mathrm{l}$ dos anticorpos primários conjugados. As incubações foram feitas com duplas ou triplas associações de anticorpos monoclonais (AcM o) específicos marcados com diferentes fluorocromos (fluoresceína - FITC ou ficoeritrina - PE).

A aquisição e análise dos casos foi procedida em citômetro de fluxo, seja no Epics-Coulter ${ }^{\circledR}$ ou no FacScan ${ }^{\circledR}$, Becton Dickinson, vigentes no setor, onde foram contadas 104 células por cada tubo. Para as análises citofluorimétricas foi utilizado um painel de AcM o capazes de reconhecer as seguintes moléculas de superfície: anti- CD 45, CD 14, CD 15, CD 34, CD 117, CD 19, CD 10, CD 20, CD 7, CD 3, CD 2, CD 4, CD 5, CD 8, CD 1a, CD 13, CD 33, CD 11b, TdT, aM P0, CD 79a, CD 61, CD 42, CD 38, H LA-D R, CD 56, CD 7.1, glicoforina-a, clg e smlg. O s AcM o utilizados foram adquiridos por diversos fabricantes como $C$ oulter, Imunotech, Becton-Dickson, Pharmigen e Dako. As marcações imunofenotípicas ao longo dos anos foram realizadas com os mesmos clones dos AcM 0 apesar de diferentes lotes de fabricação. No entanto, na mudança de lote eram realizados testes para avaliar a melhor concentração de uso para cada AcM o de forma a manter o mesmo nível da reação antígeno-anticorpo.

$\mathrm{N}$ o estabelecimento dos critérios de positividade para cada AcM o, adotamos o valor mínimo de $20 \%$ das células analisadas. O s marcadores de células precursoras como aM PO , CD 34 eT dT, por serem muito específicos e sensíveis, foram considerados positivos quando os valores excederam em $10 \%$ das células analisadas. N os casos em que havia baixa leucometria ou pouco material para estudo, a tipagem era feita por lise de hemáceas.

AN ÁLISES ESTATÍSTICAS. As análises estatísticas para avaliação de freqüências e correlações foram realizadas utilizando o programa SPSS (Versão 11.0 para $W$ indows). Foram considerados como significativos os valores de $p<0,05$.

\section{RESULTADOS}

No período de estudo, foram encaminhadas 720 amostras de M O e/ou SP para estudos imunofenotípicos que obtiveram diagnóstico de LA. Passaram pelo critério de elegibilidade os casos de LLA de células precursoras B ou T, ou LM A. A tabela 3 representa uma avaliação global dos 720 casos entre os quais foram encontradas as 60 amostras com FA, ou seja, 8,3\% dos casos de LAs entre janeiro de 2000 a novembro de 2003. As LM As foram responsáveis pela maioria dos casos com aberrações entre os anos de 2000-2002, enquanto em 2003, as aberrações imunofenotípicas foram mais encontradas em LLA. Analisando os subtipos separadamente, não encontramos diferenças estatísticas entre os grupos de LLA e LM A $(p=0,45)$ nos 28 casos de LLA e 32 de LM A com expressões aberrantes, o que em termos percentuais significa $46,67 \%$ e $53,33 \%$, respectivamente. A distribuição dos pacientes com FA, por idade, sexo e procedência é mostrada na tabela 4. A faixa etária é significativamente associada $(p=0,001)$ aos casos entre 2-16 anos de idade, onde se concentraram $85,71 \%$ dos FAs. A diferença entre os sexos foi uma variável expressiva apenas nos casos com LM A, em que os indivíduos do sexo masculino somaram quase o dobro dos casos dos do sexo feminino $(p=0,00)$. Análises com correlações regionais interestaduais, demonstraram uma tendência de aumento de expressão de CD 56 em LM As $(p=0,02)$. Analisando a freqüência detodos estes subtipos

Tabela 3 - Sumário da distribuição da amostragem total

\begin{tabular}{c|c|c|c|c|c|c|c|c}
\hline \multirow{2}{*}{ ANO } & \multicolumn{4}{|c|}{ LLA } & \multicolumn{4}{c}{ LMA } \\
\cline { 2 - 10 } & \multicolumn{2}{|c|}{ Total } & \multicolumn{2}{c|}{ Aberrantes } & \multicolumn{2}{c}{ Total } & \multicolumn{2}{c}{ Aberrantes } \\
\cline { 2 - 10 } & $n$ & $\%$ & $n$ & $\%$ & $n$ & $\%$ & $n$ & $\%$ \\
\hline 2000 & 88 & 69,84 & 6 & 42,86 & 38 & 30,16 & 8 & 57,14 \\
\hline 2001 & 175 & 66,54 & 7 & 38,89 & 88 & 33,46 & 11 & 61,11 \\
\hline 2002 & 128 & 69,19 & 5 & 35,71 & 57 & 30,81 & 9 & 64,29 \\
\hline 2003 & 104 & 71,23 & 10 & 71,43 & 42 & 28,77 & 4 & 28,57 \\
\hline Sub-Totais & 495 & 68,75 & 28 & 46,67 & 225 & 31,25 & 32 & 53,33 \\
\hline
\end{tabular}


Tabela 4 - Características de idade, sexo e procedência dos pacientes com aberração fenotípica

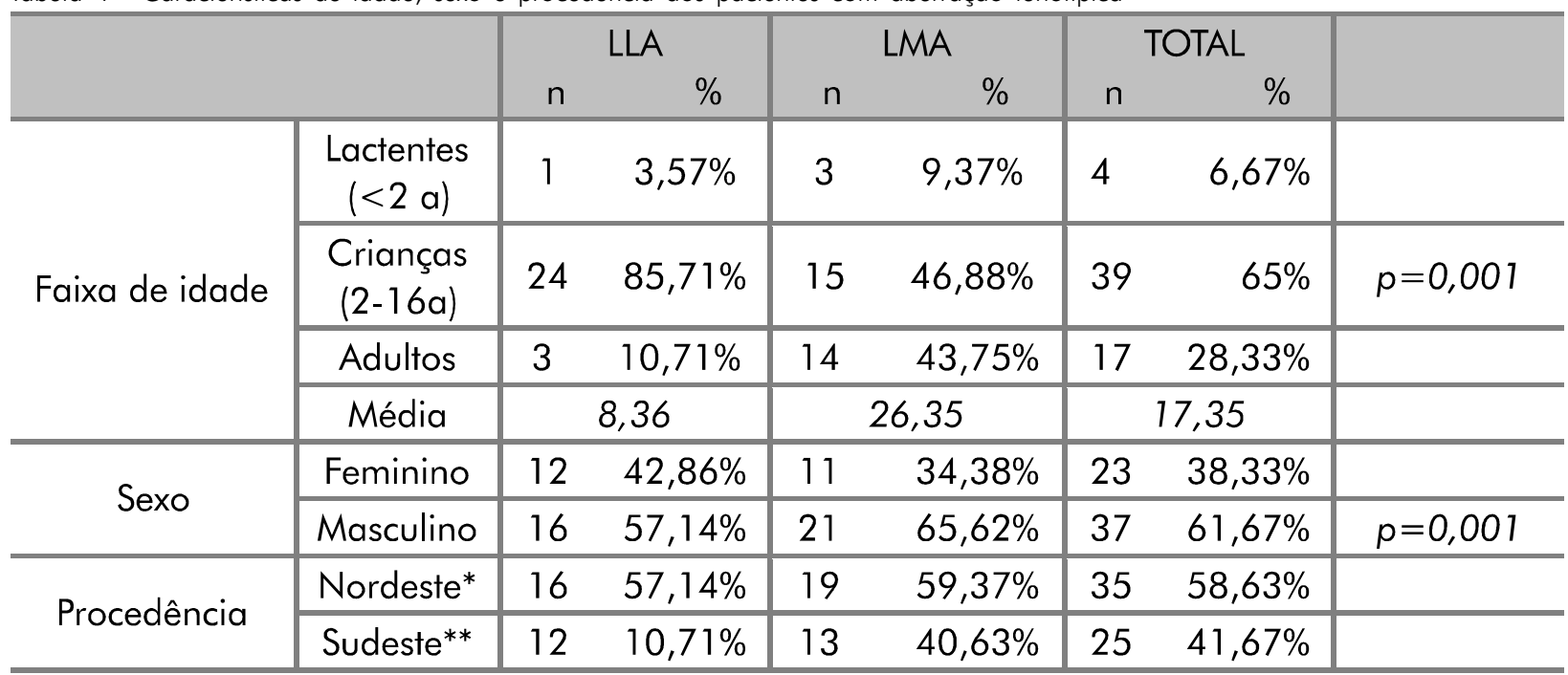

*Bahia, Paraíba e Pernambuco; **M inas Gerais e Rio de Janeiro

de LLA e LM A, ficou constatado que a LLA (CD 10+), contribuiu com o maior número $(31,67 \%)$ de amostras com imunofenótipos aberrantes. A tabela 5 explicita os resultados numéricos referentes aos subtipos das leucemias com FA. As aberrações de fenótipo encontradas foram devidas a expressão dos antígenos CD 13, CD 15 e CD 33 nos casos de LLA, e a expressão dos antígenos CD 7, CD 19 e CD 56 nos casos de LMA. A expressão do marcador linfóide CD 7 em LMA foi a mais freqüentemente encontrada ( $25 \%$ ), seguida da expressão do $\operatorname{CD} 13(31,67 \%)$ em LLA do tipo comum e (13,33\%) em LLA-T. A figura 1 exemplifica dois casos de FA, de acordo com os histogramas pela citometria de fluxo.

Tabela 5 - Distribuição dos casos quanto à aberração imunofenotípica em LLA e LMA

\begin{tabular}{c|c|c|c}
\hline Diagnóstico & $\begin{array}{c}\text { Marcador } \\
\text { aberrante }\end{array}$ & $\mathrm{n}$ & $\%$ \\
\hline LLA & CD13 & 14 & 23,33 \\
\hline LLA & CD15 & 3 & 5 \\
\hline LLA & CD33 & 11 & 18,33 \\
\hline LMA & CD7 & 20 & 33,34 \\
\hline LMA & CD19 & 5 & 8,33 \\
\hline LMA & CD56 & 7 & 11,67 \\
\hline TOTAL LMA & & 60 & 100 \\
\hline
\end{tabular}

\section{DISCUSSÃO}

0 presente estudo demonstra que a ocorrência de fenótipos aberrantes em LA é um fenômeno freqüente, observado em $8,33 \%$ dos casos. Esta freqüência se assemelha aos resultados encontrados por Saxena e cols. $1998^{12}$ que relataram a freqüência de $10 \%$ de marcadores aberrantes em estudo realizado com 60 casos e Bradstock e cols. que mostraram que, entre 568 casos de leucemias agudas, 9,6\% expressavam FA e entre as LM As $56 \%$ apresentavam marcadores linfóides ${ }^{2}$. N ão existe predomínio de freqüências entre LLA ou LMA na nossa série de casos analisados consecutivamente e com o mesmo protocolo de testes, nos levando a acreditar que nosso resultado é bem representativo quanto ao perfil das LAs na nossa população. Khalidi e cols. encontraram em $48,1 \%$ de FA nos casos de LM ALy+e $45,6 \%$ de LLA-My+ ${ }^{13,14}$.

0 fator idade parece influenciar as proporções de FA, ou seja, na faixa etária em que a LLA é mais comum, são também mais comuns os casos aberrantes e viceversa. LLA de células precursoras $B$ em casos pediátricos obteve uma freqüência maior de expressão de antígenos mielóides do que os casos de adultos ${ }^{14}$. Este perfil se traduz em maior aplicabilidade de testes para doença residual mínima nas crianças. Em relação a gêneros, a leucemia é uma doença que, de fato, acomete o sexo masculino com maior freqüência do que o sexo feminino, portanto era esperado na nossa série de casos, que maior freqüência de FA fosse encontrada entre os homens ${ }^{12,15}$. Colocando em foco as aberrações imunofenotípicas propriamente ditas, com $33,34 \%$ dos casos, a LMA/ CD 7+ocupou o primeiro lugar, seguida de $23,33 \%$ dos casos de LLA/CD 13+. N osso dado é semelhante aos de Bradstock e cols. (2), que relatam a expressão do CD 7 em $28,4 \%$ de seus casos de $L A$, porém, difere expressivamente do estudo de Khalid e cols. ${ }^{13}$, que encontraram como marcador aberrante mais freqüente 


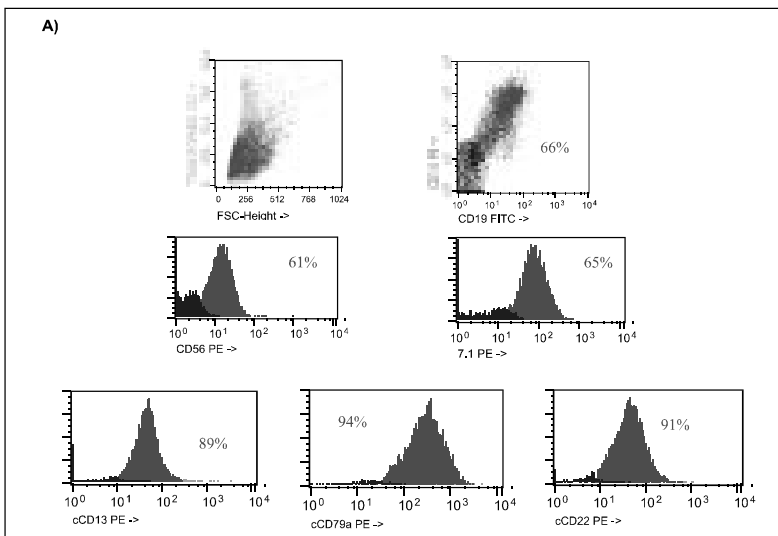

B)
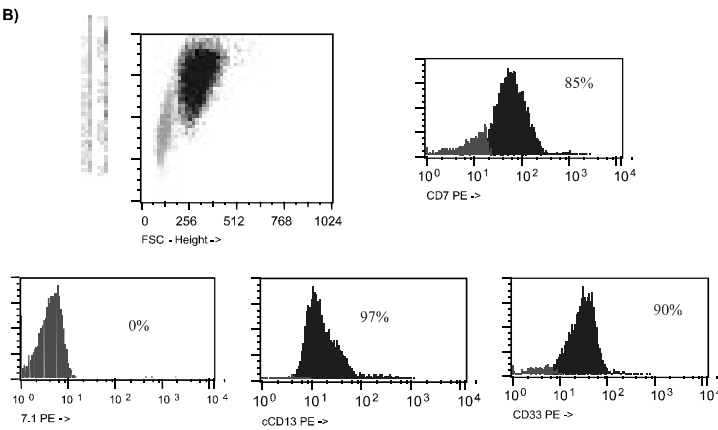

Figura 1 - Histogramas representativos da análise pela citometria de fluxo. A) Amostra de MO de LLA/CD13+. B) Amostra de MO de $\mathrm{LMA} / \mathrm{CD} 7+$

- CD20, deixando o CD 7 com a segunda posição. A expressão do $C D 7$ em LM A está associada à presença de antígenos imaturos (CD 34, H LA-DR, TdT) e, aos rearranjos do gene receptor do antígeno de diferenciaçãa de linhagens T e B (TCR e IgH). É possível que isto explique sua origem em estágios bastante indiferenciados no desenvolvimento da célula mieloblástica ${ }^{12}$. Esta explicação é também plausível de acordo com nossos resultados, pois os casos $L M A / C D 7+$ se concentraram em sua maioria entre os subtipos M 0 e M 2.

Os valores prognósticos dos FAs são parcialmente conhecidos e muitas vezes discordantes na literatura. Enquanto um estudo pediátrico de LLA M y+ sugere que este imunofenótipo não tem significância prognóstica independente ${ }^{16}$, a expressão de CD 15 é característica de LLA-B com a translocação t(4;11), sabidamente um grupo relacionado a um prognóstico ruim ${ }^{17}$. Para isto, estudos prospectivos usan do protocolos de tratamento seriam necessários para estabelecer se os pacientes com FA teriam o curso da doença diferenciado. No entanto, como em vários subtipos das LAs existe forte correlação com o genótipo, esta grande diversidade de marcadores relatados no presente estudo, poderá ser conseqüência da já referida heterogeneidade biológica da origem celular e das alterações moleculares associadas encontradas nas LAs.

Embora seja óbvio que o genótipo esteja refletido no imunofenótipo das leucemias, ainda não é absoluta a concordância na grande maioria dos casos' ${ }^{1}$. Algumas hipóteses já foram elaboradas para esclarecer a etiopatogênese das leucemias, entre elas, as que se referem as translocações do gene M LL. Em LLA, o gene AF4 no cromossomo $4 q 21$ é o gene que mais freqüentemente funde-se com o gene MLL. 0 gene M LL/AF4 aparece em aproximadamente $50 \%$ dos casos de LLA em crianças com até seis meses de idade ${ }^{19}$. Por outro lado, M LL/AF9 é mais freqüente em leucemia mielóide aguda (LM A); e MLL/ENL pode ser encontrado em LLA e LMA nas mesmas proporções'. As LLA de células precursoras $B$ com $B C R / A B L$, são tipicamente $C D 19, C D 22, T d T$, CD13 e/ou CD 33 positivos ${ }^{1}$.

Pouco se sabe sobre a relaçãa destas características imunofenotípicas anômalas e seus genótipos correspondentes em LAs. Em outras circunstâncias, a anormalidade dos fenótipos patológicos reflete assincronismos maturativos; um exemplo claro disso é uma célula B CD 19+ que ainda expressa o CD 10, característico de imaturidade, porém já apresentando CD 20 intensamente, como uma célula madura. Portanto, estas expressões aberrantes abordadas neste estudo, podem ser utilizadas na detecção de D RM e na avaliaçãa prognóstica associada a outros marcadores biológicos ${ }^{19}$.

\section{REFERÊNCIAS}

1. H rusak $O, M$ acD onald AP. Antigen expression patterns reflecting genotype of acute leukemias. Leukemia. 2002;16:1233-58.

2. Bradstock KF, KirkJ, Grimsley PG, Kabral A, H ughesWG. U nusual immunophenotypes in acute leukaemias: incidence and clinical correlations. $\mathrm{Br} J \mathrm{H}$ aematol. 1989;72:512-8.

3. Campana D, Coustan-Smith E, Janossy G. Theimmunologic detection of minimal residual diseasein acuteleukemia. Blood. 1990;76:163-71.

4. D rexler HG, Thiel E, LudwigWD . Acutemyeloid leukemias expressing lymphoid-associated antigens: diagnostic incidence and prognostic significance. Leukemia. 1993;7:489-98.

5. M acedo $A, O$ rfao $A$, Vidriales $M B$, Lopez-Berges M C, Valverde $B$, G onzalez $M$, et al. Characterization of aberrant phenotypesin acutemyeloblastic leukemia. Ann $\mathrm{H}$ ematol. 1995;70:189-94.

6. Reading CL, Estey EH , H uh YO , Claxton D F, Sanchez G, Tertappen LW, et al. Expression of unusual immunophenotype combinations in acutemyelogenous leukemia. Blood. 1993;81:3083-90. 
7. Terstappen LW, Konemann S, Safford M, Loken M R, Zurlutter K, Buchner T, et al. Flow cytometric characterization of acutemyel oid leukemia. Part 1. Significance of light scattering properties. Leukemia. 1991;5:315-21.

8. Campana D, Behm FG. Immunophenotyping of leukemia. J Immunol M ethods. 2000;243:59-75.

9. Borkhardt A, C azzaniga G, Viehmann S, Valsecchi M G, LudwigW D, Burci L, et al. Incidenceand clinical relevance of TEL/AM L1 fusion genes in children with acute lymphoblastic leukemia enrolled in the German and Italian multicenter therapy trials. Associazionel taliana Ematologica O ncologia Pediatrica and the Berlin-Frankfurt-M unster Study Group. Blood. 1997;90:571-7.

10. Baruchel A, Cayuela JM , Ballerini P, Landman-Parker J, Cezard V, Firat $\mathrm{H}$, et al. T hemajority of myeloid-antigenpositive $(\mathrm{M} y+)$ childhood B-cell precursor acutelymphoblastic leukaemias expressT EL-AM L 1 fusion transcripts. $\mathrm{BrJ} \mathrm{H}$ aematol. 1997;99:101-6.

11. Borowitz M J, Rubnitz J, N ash M , Pullen DJ, Camitta B. Surface antigen phenotype can predict TEL-AM L1 rearrengement in childhod B-precursor ALL: a Pediatric O ncology Group Study. Leukemia. 1998;12:1764-70.

12. Saxena A, Sheridan D P, C ard RT, M CPeek AM , M ewdell $C C$, Skinnider LF. Biologic and clinical significance of CD 7 expression in acute myeloid leukemia. Am J H ematol. 1998;58:278-84.

13. Khalidi H S, M edeiros LJ, Chang KL, Brynes RK, Slovak $M L$, Alber D A. The immunophenotype of adult acute myeloid leukemia: high frequency of lymphoid antigen expression and comparison of immunophenotype, $F A B$ classification, and karyotipic abnormalities. Am J Clin Pathol. 1998;109:211-20.

14. Khalidi H S, Chang KL, M edeirosLJ, Brynes RK, Slovak $M L, M$ urata-Collins J $L$, et al. Acutelymphoblastic leukemia. Survey of immunophenotype, FAB classification, frequency of myeloid antigen expression, and karyotipic abnormalities in 210 pediatric and adult cases. Am J Clin Pathol. 1999;111:467-76.

15. Sandler D P, Ross JÁ. Epidemiology of acuteleukemia in children and adults. Semin 0 ncol. 1997;24(1):3-16.

16. Pui CH , Behm FG , Singh B, Rivera GK, Schell M J, RobertsW M , et al. M yeloid-associated antigen expression lacks prognostic valuein childhood acutelymphoblastic leukemiatreated with intensivemultiagent chemoterapy. Blood. 1990;75:198-202.

17. Behm FG, Raimondi SC, Frestedt JL, Liu Q, C rist W M , $D$ owning $\mathrm{J}$, et al. Rearrangement of the $M L L$ geneconfers a poor prognosis in childhood acute lymphoblastic leukemia, regardless of presenting age. Blood. 1996;87:2870-7.

18. G olubT R, Barker GF, Bohlander SK, H iebert SW, Ward DC, Bray-Ward $P$, et al. Fusion of theT EL gene on 12p 13 to the AM L1 gene on 21q22 in acutelymphoblastic leukemia. Proc N atl Acad Sci U S A. 1995;92:4917-21.

19. Coustan-SmithE, Sancho J, H ancock M L, BoyettJM , Behm FG, Raimondi SC, et al. Clinical importance of minimal residual diseasein childhood acutelymphoblastic leukemia. Blood. 2000;96(8):2691-6. 